\title{
Impact of Second Forward-view Examination of the Right side of the Colon on Polyp and Adenoma Detection during Colonoscopy: A Randomized Controlled Trial
}

Keshu Shan

Ningbo First Hospital

Hongpeng Lu

Ningbo First Hospital

Zhixin Zhang

Ningbo University

Jiarong Xie

Ningbo University

Lu Xu

Ningbo University

Weihong Wang

Ningbo First Hospital

Lei Xu ( $\nabla$ xulei22@163.com )

Ningbo First Hospital

\section{Research Article}

Keywords: second forward-view examination, adenoma detection rate, polyp detection rate, colonic polyps, colorectal cancer, colonoscopy

Posted Date: January 18th, 2021

DOl: https://doi.org/10.21203/rs.3.rs-141084/v1

License: (9) This work is licensed under a Creative Commons Attribution 4.0 International License. Read Full License 


\section{Abstract}

Objectives: Colorectal cancer in the right side of the colon is supposed to harder to detect by colonoscopy. The aim of this study was to evaluate whether a second forward-view examination of the right side of the colon could increase adenoma detection rate (ADR) and polyp detection rate (PDR).

Methods: This was a single-centre randomized controlled trial. Patients undergoing colonoscopy were recruited and randomly assigned to the second forward-view examination (SFE) group, in which the right side of the colon was examined twice and a traditional colonoscopy (TC) group in which the colonoscopy was performed in a standard manner. The primary outcomes were the proximal PDR and ADR. The overall PDR and ADR, and advanced lesion detection rate were also recorded and compared..

Results: A total of 392 patients were included in the study (SFE group 197 vs. TC group 195). The proximal PDR and ADR in the SFE group were significantly higher than those in the TC group (PDR 17.8\% vs. $9.7 \%, P=0.021$; ADR $14.2 \%$ vs.7.2\%, $P=0.024$ ). No significant difference was found for overall $\mathrm{PDR} / \mathrm{ADR}$, or advanced lesion detection rate between the two groups.

Conclusions: This prospective study revealed that a second forward-view examination of the right side of the colon could result in a modest improvement in proximal ADR and PDR. This simple and time-effective technique might be recommended for routine colonoscopy.

Trial registration: Clinical Trials.gov, NCT03619122. Registered on 7/8/2018.

\section{Introduction}

Colorectal cancer (CRC) is one of the most commonly diagnosed malignant neoplasms worldwide[1], the incidence of which increased in recent decades[2]. Colonoscopies and endoscopic polypectomy have been proven to be an effective way to prevent CRC and decrease the mortality of CRC[3]. Thus, the adenoma detection rate (ADR) is widely accepted as an important quality indicator for colonoscopy[4]. However, many studies $[5,6]$ have reported that interval CRCs that are detected after a prior colonoscopy still account for $0.6-9 \%$ of colorectal cancers. Furthermore, several studies[7, 8] demonstrated that colonoscopy provides lower detection rate of CRCs in the proximal colon compared with that in the distal colon, which means interval CRCs are more likely to develop proximally than distally. One of the plausible theories is that polyps are easily missed due to the proximal aspect of the folds. Additionally, more flatter lesions located in the proximal colon tend to be sessile serrated adenomas which are regarded as probable precursors of $\mathrm{CRC}[9]$. Therefore, it is critical to develop a practical manipulation to improve the polyp/adenoma detection rate of the proximal colon.

A number of techniques have been implemented to achieve a potential improvement in detection of adenomas in the proximal colon, such as repeated examination, cap-assisted colonoscopy[10], and thirdrye retroscope[11]. Among these methods, a second forward-view examination of the proximal colon may be the easiest and most accessible method for endoscopists to perform, as no more equipment, staff or 
expenses are required. A prospective cohort study of 280 patients revealed additional adenomas in $15.4 \%$ of patients with an increase in the right side of the colon ADR by $6.7 \%$ [12]. Another prospective trial with 400 patients found that the increase in the ADR was $2.3 \%$ when repeated forward-view examination was performed [13]. However, these two related studies provided limited evidence supporting the implementation of a second forward-view examination of the proximal colon clinically. We therefore performed a prospective randomized-controlled trial in patients undergoing colonoscopy to evaluate whether a second forward-view examination of the proximal colon can increase proximal ADR.

\section{Methods}

\section{Study design}

A single-centre, randomized controlled trial was performed in the Endoscopy Department of Ningbo First Hospital from September 2018 to June 2019. The study protocol was approved by the Ethics Committee of Ningbo First Hospital (2018-R014) and was registered on ClinicalTrials.gov (ID: NCT03619122) on $7 / 8 / 2018$. The trial complied with the Declaration of Helsinki and written informed consent was obtained from all participants prior to inclusion. Moreover, standardized bowel manual preparation was performed for all participants. Patients who received colonoscopy were randomly assigned to the traditional colonoscopy (TC) group or second forward-view examination (SFE) group.

\section{Participants}

Outpatients (18-75 years old) who were scheduled to undergo colonoscopy at Ningbo First Hospital from September 2018 to June 2019 were recruited. Patients were excluded if they had a history of colon resection, inflammatory bowel disease or polyposis syndromes, or poor bowel preparation (Boston Bowel Preparation Scale [BBPS] score $<2$ in any segment of the colon) [14]. Participants who were unable to provide informed consent, were not successful in achieving caecal intubation, or were taking active antithrombotic therapy preventing polypectomy were also excluded.

\section{Randomization and colonoscopy procedures}

The computer-generated randomization numbers were sealed in an envelope. Included participants were randomly assigned to one of the two groups: the second forward-view examination (SFE) group, in which the proximal colon was examined twice in forward view; or the traditional colonoscopy (TC) group, in which a standardized colonoscopy procedure was performed. Complete caecal intubation was defined when the ileocecal valve and appendicular orifice were seen. The proximal colon was defined from the caecum to the hepatic flexure.

All participants received a single $2 \mathrm{~L}$ dose of polyethylene glycol (PEG) 5-6 hours before the scheduled examination time. An education video of bowel preparation was delivered to all of them by mobile phone. 
Baseline demographic characteristics including age, sex, weight, height, previous history of surgery, family history of colorectal cancer, etc. were recorded by one of the assistants prior to colonoscopy. All colonoscopies were conducted by one of four gastroenterological endoscopists who perform approximately 500-800 colonoscopies annually. High-definition colonoscopes (Olympus CF-HQ290I/CFH290I/CF-HQ290ZI, Japan) were used for all procedures.

All colonoscopies were performed without anaesthesia. Colonoscope insertion was performed in a routine manner. After successful insertion into the caecum, the scope was withdrawn to the hepatic flexure allowing the colonic mucosa to be carefully examined. At this moment, the sealed envelope with a random number was opened. If the patient was allocated to the TC group, the scope was directly withdrawn to the anus. If the patient was assigned to the SFE group, the colonoscope was advanced to the caecum again for a second inspection of the right side of the colon, and then passed to the anus. The withdrawal time was required to be at least 6 minutes. The time for the second examination was not included in the withdrawal time. Whether the patient's position was shifted during the procedure was decided by the operators.

For all endoscopies, caecal intubation time, withdrawal time, and second examination time were documented by assistants during the procedure, exclusive of therapeutic time. The adequacy of bowel preparation was scaled according to the BBPS by endoscopists. The number, size, location, and morphology of polyps were also recorded. Endoscopic polypectomy or biopsy was performed when necessary. The samples were submitted for pathological assessment. The size of the polyp was measured by visual comparison with opened forceps or snare.

\section{Statistical analysis}

Statistical analysis was carried out by using SPSS version 22.0 (SPSS Inc., Chicago, IL, USA). The sample size was calculated on the basis of a previous study. We set the proximal ADR in the traditional group at $15 \%$, and the proximal ADR in the second forward-view examination group was hypothesized to be $30 \%$. A minimal sample size of 185 participants per group would be required with a significance level of 0.05 . The statistical test used in the calculation was the two-sided pooled $Z$ test. Therefore, the investigators aimed to recruit a total of 400 participants.

The primary outcome was to compare the proximal polyp and adenoma detection rates (proximal PDR/ADR) between the two groups. The proximal ADR was defined as the proportion of patients with at least one proximal adenoma. The proximal PDR was defined as the proportion of patients with at least one proximal polyp. Secondary outcomes were overall polyp detection rate (PDR), overall ADR and advanced adenoma rate. An advanced lesion was defined as a lesion more than $10 \mathrm{~mm}$ in diameter, or with a villous component of histology or high-grade dysplasia. The advanced lesion detection rate was defined as the proportion of cases, in which more than one advanced lesion was found. In the SFE group, polyps detected on the second examination were defined as missed polyps. The numbers of polyps and adenomas per patient were also calculated. 
Continuous variables are reported as the mean and standard deviation (SD), or the median and range, according to normal or skewed distribution, respectively. Categorical variables are expressed as percentages. Unpaired Student's t-test was used to compare normally distributed continuous data; Pearson's $\chi 2$ test was used to compare categorical variables. A $p$-value $<0.05$ was considered statistically significant.

\section{Results}

\section{Baseline demographic and clinical characteristics}

We recruited 400 patients according to the inclusion criteria and randomly assigned 200 participants to each group. Five patients in the traditional colonoscopy (TC) group and 3 patients in the second forwardview examination (SFE) group were excluded due to inadequate bowel preparation (6 patients), inflammatory bowel disease (1 patient) and unwillingness to undergo polypectomy (1 patient). Finally, 392 patients were included in the analysis, of which 195 patients were allocated to the TC group, while 197 patients were allocated to the SFE group. (Fig. 1) Baseline demographic and clinical characteristics of all included patients are summarized in Table 1. 
Table 1

Baseline characteristics

\begin{tabular}{|c|c|c|c|}
\hline Parameter & $\begin{array}{l}\text { TC group } \\
(n=195)\end{array}$ & $\begin{array}{l}\text { SFE group } \\
(n=197)\end{array}$ & $P$ value \\
\hline Age (year) & $46.1 \pm 13.2$ & $47.7 \pm 12.6$ & 0.639 \\
\hline Gender(male/female) & $106 / 89$ & $107 / 90$ & 0.398 \\
\hline Weight(kg) & $62.6 \pm 11.4$ & $63.3 \pm 11.8$ & 0.337 \\
\hline Height(cm) & $165.2 \pm 8.0$ & $164.7 \pm 7.5$ & 0.494 \\
\hline $\operatorname{BMI}\left(\mathrm{kg} / \mathrm{m}^{2}\right)$ & $22.9 \pm 3.1$ & $23.2 \pm 3.5$ & 0.450 \\
\hline Indication & & & 0.416 \\
\hline Screening, n(\%) & 157(80.5) & 152(77.2) & \\
\hline Surveillance, n(\%) & $38(19.5)$ & $45(22.8)$ & \\
\hline Family history of CRC, n (\%) & $9(4.6)$ & $13(6.6)$ & 0.394 \\
\hline Previous colonoscopy, n (\%) & $50(25.6)$ & $64(32.5)$ & 0.136 \\
\hline Diabetes, n (\%) & $9(4.6)$ & 10(5.1) & 0.832 \\
\hline Hypertension, n (\%) & $25(12.8)$ & $32(16.2)$ & 0.336 \\
\hline Smoking, n (\%) & $50(25.6)$ & $51(25.9)$ & 0.955 \\
\hline Alcohol, n (\%) & $53(27.2)$ & $52(26.4)$ & 0.814 \\
\hline Intubation time (min) & $6.5 \pm 4.1$ & $6.8 \pm 3.9$ & 0.613 \\
\hline Withdrawal time (min) & $6.5 \pm 1.3$ & $6.5 \pm 1.9$ & 0.100 \\
\hline Second examination time (min) & ND & $1.4 \pm 0.6$ & ND \\
\hline Total duration of colonoscopy (min) & $13.0 \pm 4.0$ & $14.7 \pm 4.8$ & 0.208 \\
\hline Quality of bowel preparation, $\mathrm{n}$ & & & 0.884 \\
\hline BBPS 6 & 40 & 39 & \\
\hline BBPS 7 & 54 & 52 & \\
\hline BBPS 8 & 65 & 72 & \\
\hline BBPS 9 & 36 & 34 & \\
\hline \multicolumn{4}{|c|}{$\begin{array}{l}\text { Data are expressed as mean } \pm \text { standard deviation (SD), or percentage;BMI: Body Mass Index; BBPS } \\
\text { Boston Bowel Preparation Scale score. ND: No Data.TC group: Traditional Colonoscopy; SFE group } \\
\text { Second Forward-view Examination group; }\end{array}$} \\
\hline
\end{tabular}




\section{Polyps and detection rates}

For evaluation of the whole colon, a total of 155 and 134 polyps were detected in the SFE group and the TC group, respectively. The mean size of polyps was $5.26 \pm 4.46 \mathrm{~mm}$ (range $1-35 \mathrm{~mm}$ ) in the SFE group and $4.94 \pm 2.96 \mathrm{~mm}$ (range $2-20 \mathrm{~mm}$ ) in the TC group $(P=0.064)$. The mean numbers of polyps detected per patient were 0.79 [155/197] and 0.70 [134/195] in the SFE group and the TC group, respectively. The overall PDR did not differ between the two groups (SFE group 41.2\% [81/197] vs. TC group $35.9 \%$ [70/195], $P=0.288$ ). Of the polyps, there were 113 and 99 adenomas in the SFE group and TC group, respectively. The overall ADR did not differ between the two groups (SFE group 32.5\% [64/197] vs. TC group 29.7\% [58/195], $P=0.604$ ). Furthermore, advanced adenomas were found in 15 and 11 patients in the two groups, respectively. The advanced ADR presented no significant difference between the two groups (SFE group 7.6\% [15/197] vs. TC group 5.6\% [11/195], $P=0.432$ ).

\section{Proximal polyp and adenoma detection rates}

Compared with the TC group, a relatively higher proportion of proximal polyps was detected in the SFE group (34.2\% [53/155] vs. 21.6 [29/134]; $P=0.018)$. The proximal PDR showed a significant difference between the SFE group and TC group (17.8\% [35/197] vs. 9.7\% [19/195]; $P=0.021)$ (Fig. 2). The mean size of polyps found in the proximal colon was $4.92 \pm 5.66 \mathrm{~mm}$ (range 2-35 $\mathrm{mm}$ ) in the SFE group and $5.86 \pm 4.72 \mathrm{~mm}$ (range $2-20 \mathrm{~mm}$ ) in the TC group $(P=0.450)$ (Table. 2 ). The number of proximal polyps per patient in the SFE group was higher than that in the TC group $(0.27[53 / 197]$ vs. $0.15[29 / 195], P=$ $0.003)$. 
Table 2

Characteristics of proximal polyps

\begin{tabular}{|llll|}
\hline Parameter & $\begin{array}{l}\text { TC group } \\
(\boldsymbol{n}=\mathbf{2 9})\end{array}$ & $\begin{array}{l}\text { SFE group* } \\
(\boldsymbol{n}=\mathbf{5 3})\end{array}$ & $P$ value \\
\hline Polyp size, mm & $5.86 \pm 4.72$ & $4.92 \pm 5.66$ & 0.450 \\
\hline$<5 \mathrm{~mm}$ & 17 & $36(13)$ & 0.400 \\
\hline $5-9 \mathrm{~mm}$ & 8 & $15(5)$ & 0.945 \\
\hline$\geq 10 \mathrm{~mm}$ & 4 & $2(0)$ & 0.096 \\
\hline Polyp shape, $\mathrm{n}$ & 17 & $27(12)$ & 0.505 \\
\hline Is & 12 & $23(6)$ & 0.860 \\
\hline Isp & 0 & $3(0)$ & 0.192 \\
\hline Ip & & & \\
\hline $\begin{array}{l}\text { TC group: Traditional Colonoscopy; SFE group: Second Forward-view Examination group; * polyps } \\
\text { detected at second exam. }\end{array}$ & & \\
\hline
\end{tabular}

There were 28 proximal adenomas detected in the SFE group, and 14 in the TC group. The proximal ADR was significantly higher in the SFE group than in the TC group (14.2\% [28/197] vs. $7.2 \%[14 / 195] ; P=$ 0.024) (Fig. 2). The numbers of proximal advanced ADRs were similar in the two groups (SFE group 1.0\% [2/197] vs. TC group 2.1\% [4/195]; $P=0.403)$.

\section{Withdrawal time}

There was no statistically significant difference in intubation time or withdrawal time between the SFE group and the TC group ( $6.5 \pm 1.3$ minutes vs. $6.5 \pm 1.9$ minutes; $P=0.100)$; however, when the second reexamination time was included, the duration of colonoscopy was 1.7 minutes longer in the reexamination group than in the TC group, but without statistically significant difference $(14.7 \pm 4.8$ minutes vs. $13.0 \pm 4.0$ minutes; $P=0.208$ ).

\section{Discussion}

In this single-centre randomized controlled trial, we found that a second forward-view examination of the right side of the colon can significantly increase proximal PDR and ADR to $17.8 \%$ and $14.2 \%$ respectively, compared with $9.7 \%$ and $7.2 \%$, respectively, in the traditional colonoscopy group. However, there was no significant difference in the overall PDR, ADR or proximal advanced lesion detection rate between the two groups. 
Previous studies $[15,16]$ have shown that the right sided colon is associated with a lower colon cancer detection rate mainly due to certain factors, such as missed lesions, inadequate examinations, or incomplete resection. Thus, re-examination of the right side of the colon will provide a more complete colonic mucosa inspection and lower the missed adenoma rate. A randomized controlled trial conducted by Guo, et al[17] indicated that the proximal ADR in the re-examination group was higher than that in the extended withdrawal time group ( $33.1 \%$ vs. $23.6 \% ; P=0.045)$. Our primary outcome is consistent with these findings. However, there are several differences between these studies that lead to his proximal ADR (33.1\%) being much higher than ours (17.8\%). One is the definition of the proximal colon. In his study, the proximal colon was defined from the caecum to the splenic flexure, rather than from the caecum to the hepatic flexure, which was used in our study. The other is the study population. In his study, the participants were at intermediate or high risk of advanced colorectal neoplasia according to the AsiaPacific CRC screening score, as opposed to the ordinary outpatients included in our study.

Our study found that there was no significant difference in the overall PDR or ADR between the two groups. This is in line with previous studies $[12,17]$. This is because the overall PDR and ADR were already comparatively high in the TC group. Additional detection of proximal polyps and adenomas in the second examination made overall PDR and ADR higher than those in the TC group, but did not reach a statistically significant difference (overall PDR: $41.6 \%$ vs. $35.9 \%, P=0.245$; overall ADR: 32.5 vs. $19.7, P=$ 0.418). However, the detection rate of advanced adenoma was higher in the TC group than in the SFE group, but there was no significant difference $(P=0.408)$. It is hypothesized that the sample of our trial was small and that large lesions were only detected in 4 and 2 patients in each group. Therefore, more studies with large samples are required to explore this effect.

In the present study, among the proximal polyps detected at the second exam, $72.2 \%$ were less than $5 \mathrm{~mm}$, and others were between $5 \mathrm{~mm}$ and $9 \mathrm{~mm}$. Divided by shape, $66.7 \%$ were of sessile morphology, and the others were slightly elevated. These characteristics are consistent with those described in previous studies $[18,19]$. One explanation is that the colonic mucosa of the right side forms deep and large folds. When the small flat lesions are located behind these folds, they can go easily undetected. The second examination inspected the mucosa twice carefully. Furthermore, reinsertion may stimulate the movement of the mucosa and performers can view the mucosa from different directions.

Withdrawal time is also considered to be one of the quality indicators of colonoscopy. Compared with less than 6 minutes, a withdrawal time of more than 6 minutes is associated with a higher ADR[20] and a decreased risk of interval $\mathrm{CRC}[21]$. Thus, a mean withdrawal time $>6$ minutes was guaranteed in the present study, and there was no significant difference between the two groups. When the re-examination time was included, the mean total duration of colonoscopy was 1.7 minutes longer than that of the traditional colonoscopy group, but the difference was still not statistically significant $(14.7 \pm 4.8$ minutes vs. $13.0 \pm 4.0$ minutes; $P=0.208$ ). Therefore, we believe that second forward-view examination of the right side of the colon is time effective. 
The present results provide new evidence that second forward-view examination of the right side of the colon could increase proximal adenoma and polyp detection during colonoscopy. The second examination is also time effective. Considering that there is substantial colonoscopic work to be done with high quality in many Chinese endoscopic centres, and a lack of additional training or specific equipment in rural hospitals, repeated forward-view examination of the right side of the colon is a simple, effective and readily available technique to obtain a modest improvement in the proximal ADR.

The current study has some limitations. First, the study was a single-centre study, and the sample size was small. A multicentre study with more participants will be required in the future. Second, the endoscopist was not blinded to the protocol. Hence, there was potential psychological effect on attention to lesion detection during the first and second examinations. Maybe two different endoscopists performing the first and second examinations could address this problem, but these resources are not accessible in the real world. Third, the present study did not elaborate sessile serrated adenoma/polyp (SSA/P). The biological behavior of polyps may be another plausible explanation for why colonoscopy is less effective in the right side of the colon. SSA/P is regarded as more likely to become malignant. Further studies should pay more attention to SSA/P.

\section{Conclusions}

In conclusion, this prospective controlled study revealed that a second forward-view examination of the proximal colon could increase proximal ADR and PDR. This simple and time-effective technique might be recommended for routine colonoscopy.

\section{Declarations}

\section{Ethics approval and consent to participate}

This clinical study was conducted in accordance with the guidelines of the Declaration of Helsinki. Written informed consent had been obtained from all participants prior to inclusion in the study. The study protocol was reviewed and approved by the ethics committee of Ningbo first hospital and each participating institution (reference number 2018-R014). The study was registered with ClinicalTrials.gov (ID: NCT03619122) on 7/8/2018.

\section{Consent for publication}

Not applicable.

\section{Availability of data and materials}


The datasets used and/or analysed during the current study are available from the corresponding author on reasonable request.

\section{Competing interests}

The authors declare that they have no competing interests.

\section{Funding}

This work was supported by the Medical Health Science and Technology Project of Zhejiang Provincial Health Commission (No. 2017KY581 to Lei Xu). The Zhejiang Provincial Health Commission had no role in study design, data collection and analysis, decision to publish, or preparation of the manuscript.

\section{Author's contribution}

Study concept and design: KS, LX, WW; Acquisition of data: KS, HL, ZZ, JX, LX; Analysis and interpretation of data: KS, ZZ, JR, LX; Drafting of the manuscript: KS; Intellectual content: WW, HL, JR, ZZ. All authors have read and approved the manuscript.

\section{Acknowledgments}

The authors would like to thank all participants without whom this study was impossible.

\section{Authors' information}

${ }^{1}$ China Department of Gastroenterology, Ningbo First Hospital, Ningbo 315010, China; ${ }^{2}$ College of Medicine, Ningbo University, Ningbo 315010, China

\section{References}

1. Siegel RL, Miller KD, Jemal A: Cancer statistics, 2019. CA: A Cancer Journal for Clinicians 2019, 69(1).

2. Arnold M, Sierra MS, Laversanne M, Soerjomataram I, Jemal A, Bray F: Global patterns and trends in colorectal cancer incidence and mortality. Gut 2017, 66(4):683-691.

3. Zauber A G WSJ, O"Brien M J , et al.: Colonoscopic Polypectomy and Long-Term Prevention of Colorectal-Cancer Deaths. New England Journal of Medicine 2012, 366(8):687-696.

4. Millan MS, Gross P, Manilich E, Church JM: Adenoma Detection Rate: The Real Indicator of Quality in Colonoscopy. Diseases of the Colon \& Rectum 2008, 51(8):1217-1220. 
5. Cooper GS, Xu F, Sloan JSB, Schluchter MD, Koroukian SM: Prevalence and predictors of interval colorectal cancers in Medicare beneficiaries. Cancer 2012, 118(12):0-0.

6. Martínez ME, Baron JA, Lieberman DA, Schatzkin A, Lanza E, Winawer SJ, Zauber AG, Jiang R, Ahnen DJ, Bond JH: A Pooled Analysis of Advanced Colorectal Neoplasia Diagnoses After Colonoscopic Polypectomy. 2009, 136(3):832-841.

7. Singh H, Nugent Z, Mahmud SM, Demers AA, Bernstein CN: Predictors of Colorectal Cancer After Negative Colonoscopy: A Population-Based Study. American Journal of Gastroenterology 2010, 105(3):663-673.

8. Brenner H, Hoffmeister M, Arndt V, Stegmaier C, Altenhofen L, Haug U: Protection From Right- and Left-Sided Colorectal Neoplasms After Colonoscopy: Population-Based Study. Journal of the National Cancer Institute 2010, 102(2):89-95.

9. Bettington M, Walker N, Clouston A, Brown I, Leggett B, Whitehall V: The serrated pathway to colorectal carcinoma: current concepts and challenges. Histopathology 2013, 62(3):367-386.

10. Jain D, Sandhu N, Singhal S: New Developments in Mechanical Enhancement of Colonoscopy: Cuffs, Caps and Rings. Digestion 2016, 93(3):234-247.

11. Leufkens AM, DeMarco DC, Rastogi A, Akerman PA, Azzouzi K, Rothstein RI, Vleggaar FP, Repici A, Rando G, Okolo PI et al: Effect of a retrograde-viewing device on adenoma detection rate during colonoscopy: the TERRACE study. Gastrointest Endosc 2011, 73(3):480-489.

12. Clark BT, Parikh ND, Laine L: Yield of repeat forward-view examination of the right side of the colon in screening and surveillance colonoscopy. Gastrointest Endosc 2016, 84(1):126-132.

13. Chandran S, Parker F, Vaughan R, Mitchell B, Fanning S, Brown G, Yu J, Efthymiou M: Right-sided adenoma detection with retroflexion versus forward-view colonoscopy. Gastrointest Endosc 2015, 81(3):608-613.

14. Parmar R, Martel M, Rostom A, Barkun AN: Validated Scales for Colon Cleansing: A Systematic Review. Am J Gastroenterol 2016, 111(2):197-204; quiz 205.

15. le Clercq CM, Bouwens MW, Rondagh EJ, Bakker CM, Keulen ET, de Ridder RJ, Winkens B, Masclee AA, Sanduleanu S: Postcolonoscopy colorectal cancers are preventable: a population-based study. Gut 2014, 63(6):957-963.

16. Shergill AK, Conners EE, McQuaid KR, Epstein S, Ryan JC, Shah JN, Inadomi J, Somsouk M: Protective association of colonoscopy against proximal and distal colon cancer and patterns in interval cancer. Gastrointest Endosc 2015, 82(3):529-537.e521.

17. Guo C-G, Zhang F, Ji R, Li Y, Li L, Zuo X-L, Li Y-Q: Efficacy of segmental reexamination of proximal colon for adenoma detection during colonoscopy: a randomized controlled trial. Endoscopy 2017, 49(03):243-250.

18. Desai M, Bilal M, Hamade N, Gorrepati VS, Thoguluva Chandrasekar V, Jegadeesan R, Gupta N, Bhandari P, Repici A, Hassan $\mathrm{C}$ et al: Increasing adenoma detection rates in the right side of the colon comparing retroflexion with a second forward view: a systematic review. Gastrointest Endosc 2019, 89(3):453-459.e453. 
19. Ahn SB, Han DS, Bae JH, Byun TJ, Kim JP, Eun CS: The Miss Rate for Colorectal Adenoma Determined by Quality-Adjusted, Back-to-Back Colonoscopies. Gut Liver 2012, 6(1):64-70.

20. Barclay RL, Vicari JJ, Doughty AS, Johanson JF, Greenlaw RL: Colonoscopic Withdrawal Times and Adenoma Detection during Screening Colonoscopy. N Engl J Med 2006, 355(24):2533-2541.

21. Shaukat A, Rector TS, Church TR, Lederle FA, Kim AS, Rank JM, Allen JI: Longer Withdrawal Time Is Associated With a Reduced Incidence of Interval Cancer After Screening Colonoscopy. Gastroenterology 2015, 149(4):952-957.

\section{Figures}

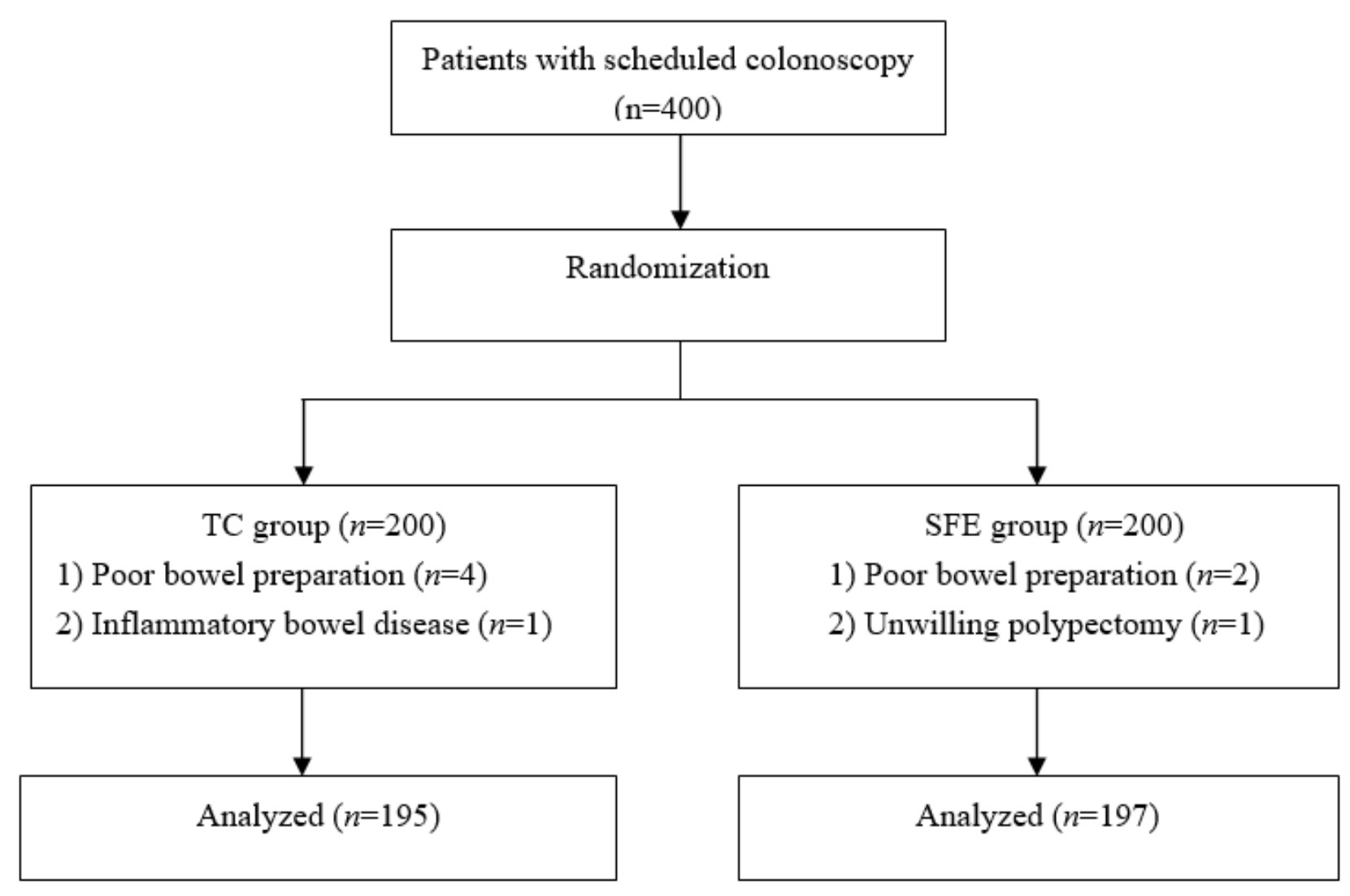

Figure 1

Flow chart for the patients. A total of 400 patients were enrolled and assigned randomly to TC/SFE group. 


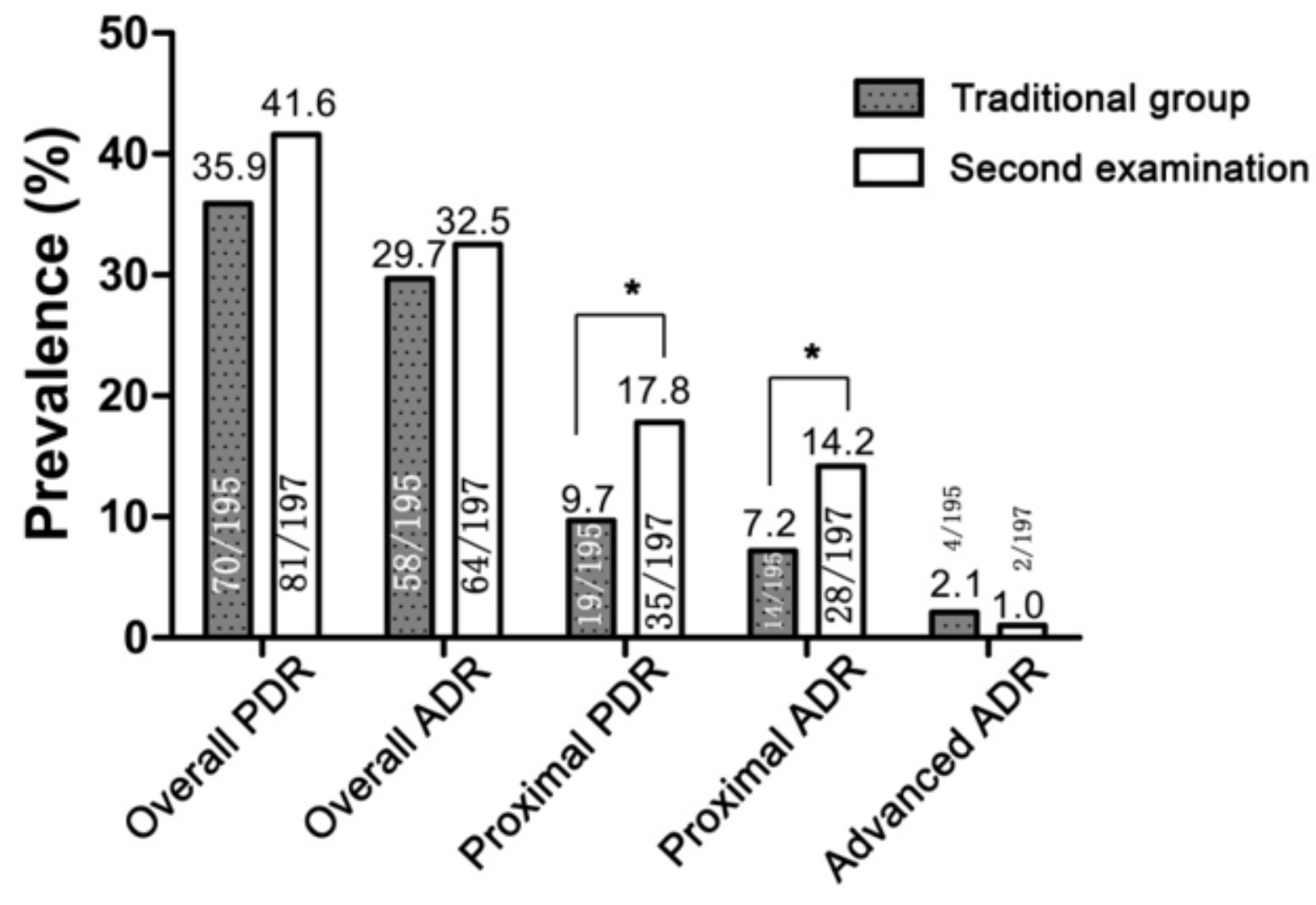

Figure 2

Polyp detection rate and adenoma detection rate. PDR polyp detection rate, ADR adenoma detection rate. Asterisks indicate $\mathrm{P}$ value $<0.05(0.021 / 0.024$ by the $\chi 2$ test in proximal PDR/ADR). $\mathrm{P}$ value $=$ $0.245 / 0.604 / 0.403$ by the $\chi 2$ test in overall PDR/ADR and advanced ADR, respectively. 